\title{
LAS PARADOJAS DEL COMUNITARISMO
}

uisiera, a modo de introducción, mencionar algunas condiciones que, a mi juicio, debe cumplir una discusión filosófica fecunda entre las posiciones comunitarista y liberal.

Como en cualquier otro ámbito o disciplina, una discusión sólo puede ser fructífera si es racional, y una discusión sólo puede ser racional si ambas partes comparten algún conjunto de creencias. Es imposible discutir cuando no hay absolutamente nada en común. Cuando éste es el caso, la discusión (o intercambio de ideas) se asemeja a lo que, en términos de Kuhn, podríamos llamar una lucha entre paradigmas. Nótese que hasta aquí mi afirmación quiere ser condicional: si se desea una discusión racional, entonces deben compartirse algunas creencias. No dice que haya o que sea posible un tal tipo de discusión. Podría ser cierto que todas las discusiones filosóficas (o científicas) fueran luchas de paradigmas. No voy a entrar en este tema. Lo que voy a hacer es, en cambio, presuponer que es posible una discusión racional entre comunitaristas y liberales.

De lo que dije hasta aquí resultan las dos primeras creencias que voy a presuponer que ambas posiciones aceptan en común: que una discusión racional presupone un acuerdo en algunas creencias, y, además, que es posible en este caso una discusión racional. La pregunta siguiente es, cuáles pueden ser esas creencias comunes en el caso particular que nos ocupa. No detallaré exhaustivamente alguna

${ }^{1}$ Agradezco a los integrantes del seminario sobre comunitarismo que, bajo la coordinación de Ernesto Garzón Valdés, tuvo lugar en la Universidad de Mainz en el semestre de verano de 1993, así como a los participantes de las Jornadas sobre comunitarismo realizadas en Tossa de Mar (España) en mayo de 1994. También le agradezco a Michael Baurmann por sus comentarios críticos. 
posible lista, pero sí señalaré dos ámbitos en que es, a mi juicio, imprescindible el acuerdo. El primero se refiere al concepto de coherencia lógica. Supondré (tercera presuposición) que tanto el comunitarismo como el liberalismo consideran que la coherencia es una condición necesaria para la aceptabilidad de una teoría moral. Esto implica aceptar la lógica estándar, y, eventualmente, sus extensiones. Simultáneamente, implica rechazar algunas «lógicas alternativas» (aquellas que niegan alguno de los principios lógicos) ${ }^{2}$. Por último (cuarta presuposición), supondré que ambas posiciones aceptan que otra condición necesaria para la aceptabilidad de una teoría moral es su compatibilidad con afirmaciones fácticas que se consideran verdaderas, o al menos confirmadas.

La tarea que me propongo en este trabajo consiste, en algún sentido, en una búsqueda. La búsqueda de lo que podríamos denominar un comunitarista coherente y genuino. Es decir, una teoría comunitarista que no contenga afirmaciones contradictorias, y que sea distinguible de cualquier otra teoría moral.

Gran parte de los desarrollos teóricos de los comunitaristas se dirigen a analizar críticamente la posición antagónica arquetípica, es decir, el liberalismo (particularmente en su versión kantiana). Por ello, creo que conviene buscar en esas críticas el origen de las diversas formulaciones teóricas que el comunitarismo puede adquirir.

La primera pregunta que creo conveniente realizar, en tomo de la crítica comunitarista al liberalismo, es cuál es exactamente el blanco de la crítica. El liberalismo, como cualquier corriente de pensamiento y acción, posee una multiplicidad de facetas, y diferentes críticas pueden estar dirigidas a diferentes aspectos, que conviene siempre diferenciar. Michael Walzer, por ejemplo, distingue dos blancos de ataque ${ }^{3}$. Por un

${ }^{2}$ Esto parece muy inofensivo, pero no lo es tanto, si prestamos atención a afirmaciones como la siguiente: «...the tragic protagonist (aquél que caracteriza la típica situación de los agentes morales, según MacIntyre) cannot do everything that he or she ought to do. This "ought", unlike Kant's, does not imply "can". Moreover any attempt to map the logic of such "ought" assertions on to some modal calculus so as to produce a version of deontic logic has to fail». (MacIntyre [1985], p, 224). Y lo es todavía menos, si recordamos que al menos algunos comunitaristas toman aspectos esenciales de la filosofía de Hegel. Si la discusión entre comunitaristas y liberales ha de ser encarada como una reiteración de la discusión entre kantianos y hegelianos, entonces creo que estamos condenados a un diálogo entre sordos.

${ }^{3}$ Walzer (1990a), esp. págs. 7-11. Invierto el orden en que Walzer presenta sus dos críticas. 
lado, el liberalismo en su faz teórica, es decir, la teoría política, o filosófico-política liberal, y, por otro, el liberalismo en su faz práctica, es decir, las sociedades liberales realmente existentes; llamaré a esto último, siguiendo a Walzer, la práctica liberal. Como bien lo indica Walzer, esta distinción da lugar a dos críticas que, al menos a primera vista, parecen ser algo contradictorias entre sí. Por un lado, se le dirige una crítica directamente a la teoría liberal. Esta crítica le reprocha a la teoría liberal haber construido un modelo de individuo o agente moral excesivamente abstracto. Sandel denomina a este yo liberal «yo trascendental»o «unencumbered self», refiriéndose a las dos versiones en su opinión más importantes de la teoría liberal: la teoría kantiana y la teoría de Rawls ${ }^{4}$. Se trata de un yo moral que no se condice con la realidad moral o psicológica de los individuos de carne y hueso. Por otro lado, se le dirige una crítica a la práctica liberal, es decir, a las sociedades liberales, según la cual esta práctica ha creado individuos (reales) excesivamente egoístas, separados entre sí, sin vínculos, carentes de solidaridad, de ideales comunes, etc. Indirectamente, esta crítica se dirige también a la teoría liberal, en la medida en que ésta refleja fielmente una práctica moral que no es deseable, y de la que, incluso, es parcialmente responsable. Según la primera crítica, la teoría liberal no refleja adecuadamente la realidad moral de los seres humanos en sociedad, ni siquiera los que pertenecen a sociedades liberales; según la segunda, en cambio, la teoría liberal sí refleja adecuadamente la realidad moral de los seres humanos en las sociedades liberales, es decir, la práctica liberal. Sólo que esta práctica tiene consecuencias morales no deseables. Dejo de lado el hecho de que estas dos críticas resultan (por lo menos) conflictivas entre sí, y de qué modo podría encontrarse un compromiso entre ambas.

Me interesa en cambio realizar otra distinción dentro del liberalismo, que da lugar a dos críticas similares a las mencionadas por Walzer, pero no idénticas. Esta distinción se sitúa dentro de la teoría liberal misma. Es posible, creo, diferenciar en la teoría moral (o conjunto de teorías morales) que denominamos «liberal», dos niveles (una distinción análoga es, creo, aplicable a cualquier teoría moral):

1. Por un lado, un nivel que (sin pretender excesivo rigor terminológico) denominaré «metaético» o de fundamentación. En este nivel, me interesa distinguir dos tesis liberales. En primer lugar, una tesis que llamaré «universalismo o tesis universalista», según la cual es posible fundamentar, o justificar normas morales universales,

\footnotetext{
${ }^{4}$ Ver Sandel ( 1984), esp. pág. 85.
} 
donde «universales» quiere decir, que poseen una validez que trasciende las diferentes concepciones del bien o fines particulares de tal o cual sociedad o individuo. En segundo lugar, una tesis que llamaré «neutralismo» o «tesis neutralista», según la cual es posible fundamentar o justificar normas morales neutrales respecto de las diferentes concepciones del bien o fines particulares de un individuo o sociedad; estas normas neutrales son, obviamente, las normas liberales sustantivas.

Me interesa detenerme con algún detalle en estas dos tesis liberales, para observar con precisión sus diferencias. Que existen normas cuya validez es universal significa, específicamente, que su obligatoriedad moral no depende de ningún contexto social o geográfico. Su obediencia es moralmente obligatoria para cualquier individuo, cualquiera que fuera su contexto o su concepción del bien. Obviamente, las normas morales universales son todas, en un cierto sentido, neutrales, porque valen independientemente de las concepciones del bien y los fines individuales o sociales. Pero no es éste el sentido de «neutral» al que se refiere la segunda tesis, que he denominado «tesis neutralista». Para el liberalismo, es posible fundamentar ciertas normas que, además de ser universalmente válidas, son independientes, es decir, no están conceptualmente vinculadas a ninguna concepción del bien. Su contenido (no su validez u obligatoriedad) no se ve «contaminado» con ninguna concepción del bien. Si la tesis neutralista es verdadera, entonces quiere decir que, al menos en ciertas circunstancias, es posible para un agente moral distanciarse de su concepción del bien (es decir, de su medio social, sus fines, su tradición, etc.) y reflexionar moralmente con independencia de esos contextos. Esto no está implicado necesariamente en la tesis universalista.

Es probable que, agregando ciertas premisas adicionales, sea posible encontrar una relación inferencial entre la tesis universalista y la tesis neutralista. Desde el punto de vista del liberalismo, es obvio que la neutralidad conforma una razón para creer que las normas poseen validez universal; además, para el liberal, una norma sólo podrá poseer validez universal si su contenido no depende de ninguna concepción del bien o tradición particular. Pero esta inferencia no es necesaria para los fines que me propongo aquí. De hecho, es al menos concebible una teoría que defienda una determinada concepción del bien y, simultáneamente, sostenga que ella posee una validez universal ${ }^{5}$. Del mismo modo, lo es, al menos como posibili-

${ }^{5}$ Ejemplos de teorías universalistas que no defienden la tesis neutralista podrían ser el marxismo, o algún tipo de perfeccionismo. La pretensión de imponer una determinada religión universalmente puede ser también interpretada de este modo. 
dad lógica, una teoría cuyo contenido fuera neutral, pero que no fuera moralmente vinculante para cualquier individuo en cualquier sociedad o contexto cultural. Lo importante es que el liberalismo necesita y sostiene ambas tesis, y que ambas serán blanco de crítica comunitarista.

2. Por otro lado, tenemos el nivel «normativo» o sustantivo, es decir, aquel en el que se da el conjunto mismo de normas o valores «liberales». Aquí se sitúa lo que llamaré la «teoría liberal sustantiva»: los derechos individuales, la tolerancia religiosa, la libre expresión, las normas de justicia distributiva, el valor de la autonomía individual, la democracia liberal, etc.

Teniendo en cuenta esta distinción, pueden surgir dos críticas comunitaristas frecuentes, que, como dije, se relacionan con las dos críticas que menciona Walzer, sólo que ahora ambas están dirigidas a estos dos diferentes aspectos de la teoría liberal:

1. En primer lugar, se suele dirigir una crítica al aspecto metaético del liberalismo. Ésta se dirige a las dos tesis liberales que he distinguido en este nivel. Por un lado, se critica la tesis neutralista: sostener que pueden fundamentarse normas morales (es decir, las normas liberales sustantivas) de un modo neutral respecto de cualquier concepción del bien presupone un modelo de agente moral totalmente abstracto, desligado de toda circunstancia social concreta o particular. El problema no es aquí, como en la primera crítica de Walzer, que este agente sea «irreal», es decir, que no se condiga con la práctica liberal. Este modelo de agente moral es criticable ya en el plano de la teoría, porque carece de las notas esenciales que hacen posible la moralidad misma, básicamente, la inclusión dentro de un marco narrativo, historia o tradición determinada, sea cual fuera esta historia o tradición. Por otro lado, también se critica el universalismo liberal, sosteniendo que la inclusión en un contexto o tradición es la fuente de legitimidad de las normas morales. Una norma sólo puede ser vinculante si emana del marco comunitario al que pertenezco. Esto quiere decir que la obligatoriedad de una determinada concepción moral no puede extenderse de un modo universal. Los dos aspectos de la crítica (i. e., al neutralismo y al universalismo) están, naturalmente, muy vinculados y no aparecen diferenciados en la argumentación comunitarista.

2. En segundo lugar, se dirige una crítica a la teoría liberal sustantiva, por defender valores excesivamente individualistas, promover el egoísmo, la aparición de «free-riders», desincentivar virtudes comunitarias como el patriotismo, la solidaridad, etc. 
La relación entre estas dos críticas parece, a primera vista, ser no tan conflictiva como la de las dos críticas mencionadas por Walzer. Por el contrario, podría hasta encontrarse un vínculo argumentativo, que podría ser el siguiente: el modelo de agente moral que propone la teoría liberal metaética es un yo moral descarnado, neutral, «egoísta racional», capaz de desligarse de todo vínculo comunitario para elegir sus fines, que por tanto carece de las notas esenciales de un verdadero agente moral, y da lugar, naturalmente, a normas y valores sustantivos vaciados de autoridad moral. Sin embargo, la primera crítica es, en un sentido, bastante más débil que la segunda, y, si bien es posible que la segunda presuponga a la primera (o al menos alguna interpretación de la primera), la inversa no necesariamente debe darse. Creo, por otro lado, que ambas críticas reflejan posiciones teóricas profundamente diferentes dentro del comunitarismo. Por ello, me detendré algo más en analizar cada una de ellas:

1. La primera crítica creo que pertenece de modo arquetípico a toda posición comunitarista. Sostiene, fundamentalmente, lo siguiente: el liberalismo pretende ser una teoría «neutral» respecto de las concepciones del bien, o valoraciones particulares, fines, etc. Es decir, pretende no presuponer ninguna concepción del bien determinada, sino sólo establecer un marco en el que diversos ideales del bien puedan desarrollarse. Pero esto es (de acuerdo con esta crítica comunitarista) una pretensión imposible, que, incluso, frustra la posibilidad misma de la moralidad, que siempre aparece vinculada con una determinada concepción del bien. Es plausible pensar que la razón para rechazar el universalismo liberal sea este rechazo al neutralismo (aunque no deseo abrir juicio al respecto). Cierto es que la imposibilidad de distanciarse y ubicarse en un punto de vista neutral hace difícil pensar que una determinada concepción moral pueda valer universalmente. Así como su fundamentación y contenido, también su obligatoriedad surge de un modo particular. En este sentido, el comunitarismo encarna de modo arquetípico la defensa de un particularismo moral, es decir, de la negación del universalismo.

Además, continúa esta crítica, esto ha contribuido a alimentar, dentro de la práctica liberal, una autocomprensión equivocada y peligrosa: que la propia teoría y práctica están más allá de toda tradición, o, más bien, que carece de tradición; que sus valores son universales y neutrales, y no dependen de ninguna circunstancia históricas o social concreta. MacIntyre, por ejemplo, expresa esta opinión, del siguiente modo:

«When men and women identify what are in fact their partial and particular causes too easily and too completely with 
the cause of some universal principie, they usually behave worse than they would otherwise do» ${ }^{6}$. (Aquí se ve claramente el carácter antiuniversalista de la posición de MacIntyre).

De modo más concreto, también Walzer sostiene que esta pretensión universalista ha tenido y tiene consecuencias nefastas, tales como la justificación del imperialismo y el sojuzgamiento de naciones supuestamente «bárbaras» o «atrasadas» ${ }^{7}$. El universalismo liberal (que él incluye dentro de lo que denomina «covering-law universalism»), dice Walzer, es «always a cover for expansion and exploitation»; o, utilizando de una metáfora teológica: «Covering-law universalism is a jealous God, and all other gods but mine are idols».

Pero téngase en cuenta que esta crítica no dice nada acerca de cuál deba ser el contenido de la concepción del bien concreta que una comunidad posee o debe poseer. Más aún, de esta crítica se desprende que la teoría liberal misma, en su nivel sustantivo, refleja una concepción del bien, y la práctica liberal constituye una tradición como cualquier otra. Esta concepción del bien habla, entre otras cosas, de los derechos individuales, del valor de la autonomía, de la libertad para perseguir planes de vida diversos (dentro de ciertos límites), etc. Lo único que dice esta primera crítica es que la teoría liberal se equivoca cuando afirma ser neutral respecto de valores o concepciones del bien particulares y que las normas liberales poseen una validez universal.

Ahora bien, si es cierto que la aspiración universalista y neutralista del liberalismo es imposible, entonces quiere decir que estamos «atados» a determinado conjunto de valores e ideales, del cual no podemos tomar distancia y juzgarlo neutralmente. El propio marco es, para usar un término frecuente en los comunitaristas, «inescapable», porque nos constituye como agentes morales. Taylor expresa esta idea claramente:

«...our discussion on identity indicates rather that it belongs to the class of the inescapable, i. e., that it belongs to human agency to exist in a space of questions about strongly valued goods, prior to all choice or adventitious cultural change» ${ }^{8}$.

Esto implica, entonces, necesariamente, que alguien perteneciente a una sociedad liberal (que defiende los valores de la teoría liberal sustantiva) tampoco podrá tomar distancia, y deberá ser, por lo tanto,

${ }^{6}$ MacIntyre (19851, pág. 221.

${ }^{7}$ Walzer (1990b), pág, 541.

${ }^{8}$ Taylor (1989), pág. 31. 
liberal. Las normas liberales sustantivas serán, además, genuinamente vinculantes sólo para aquellos que pertenecen al marco liberal. No debería ser, por tanto, sorprendente que esto les ocurra a los propios comunitaristas. De hecho, es cierto que ninguno de los principales comunitaristas desea abandonar, por ejemplo, el discurso de los derechos individuales, o los valores democráticos, ninguno desea caer en un conservadurismo preliberal. Walzer expresa claramente esta idea diciendo:

«The language of individual rights -voluntary association, pluralism, toleration, separation, privacy, free speech, the career to talents, and so on- is simply inescapable. Who among us seriously attempts to escape? If we are situated selves $[\ldots]$ then our situation is largely captured by that vocabulary» ${ }^{9}$.

Dicho de otro modo, Walzer viene a decir algo así como: si esta crítica comunitarista es correcta, es decir, si es cierto que la teoría liberal metaética es falsa, y nuestra identidad está determinada por el marco o tradición al que pertenecemos y es cierto que el liberalismo es una tradición, entonces nosotros, que pertenecemos a la tradición liberal, deberemos ser, al menos en gran medida, liberales. Dicho burdamente, ser comunitarista en el plano metaético, en una comunidad liberal, implica ser liberal en el plano sustantivo.

2. La segunda crítica lleva a una posición sustancialmente más fuerte. Es más fuerte porque no solamente critica al liberalismo en su faceta metaética (es decir, al neutralismo y universalismo liberal), sino también en su faceta sustantiva. No dice solamente que la teoría liberal metaética, sino incluso la teoría liberal sustantiva, es decir, los valores o normas liberales, son rechazables. ¿Por qué los valores liberales son rechazables? Porque, si bien puede ser cierto que el liberalismo constituye, como lo afirma la primera crítica (y contrariamente a lo que afirma la teoría liberal metaética), una tradición y un marco comunitario como cualquier otro, la teoría liberal sustantiva ha contribuido a que este marco no sea suficientemente homogéneo. El liberalismo es una tradición, pero demasiado débil. Aquí las ideas frecuentemente defendidas por el comunitarismo de que la moralidad presupone una idea del bien, y de que la identidad como agentes morales presupone un marco comunitario, tienen un significado diferente y mucho más fuerte que en el caso de la primera crítica. Lo que se defiende en este caso es la necesidad de una concepción

${ }^{9}$ Walzer (1990b), pág. 14. 
del bien y del agente moral comunitaria, es decir, en la que prevalezcan valores o virtudes como la solidaridad, y los vínculos familiares, vecinales o nacionales. Se considera la justicia sólo como un remedio indeseable, un mal menor, cuando una comunidad está enferma y carece de verdaderas virtudes. Al respecto, es paradigmática la posición de Sandel al referirse al problema de las circunstancias de justicia dentro de la teoría de Rawls. Las circunstancias de justicia de carácter subjetivo consisten básicamente en el altruismo limitado y el egoísmo desinteresado (i. e. no envidioso). Si estas circunstancias se dan, dice Sandel, entonces es lógicamente necesaria la justicia. Pero estas circunstancias se dan, precisamente, cuando otras virtudes, como las que he mencionado, no están presentes. Para ilustrar este argumento, Sandel menciona el caso de la familia. En una familia en la que existen lazos afectivos fuertes y reina relativa armonía, predomina la generosidad y nadie está constantemente detrás de aquello que, según criterios de justicia, le corresponde. En la medida que esta armonía decrece y los conflictos aumentan, el afecto y la espontaneidad ya no son efectivos y debe recurrirse a la justicia y a la observancia de los derechos de cada uno $^{10}$. ¿Significa esto que se ha producido un mejoramiento moral? De ningún modo; por el contrario, afirma Sandel:

«If an mercase in justice does not necessarily imply an unqualified moral improvement, it can also be shown that in some cases, justice is not a virtue but a vice ${ }^{11}$.

Esto es coincidente con la visión de Hume, que Sandel cita precisamente en este contexto:

«Increase to a sufficient degree the benevolence of men, or the bounty of nature, and you render justice useless, by enpplyin its place with much nobler virtues, and more favourable blessings ${ }^{12}$.

Si esta crítica es correcta, entonces no se trata solamente de que una sociedad defienda una concepción del bien cualquiera, sino que, insisto, debe ser una concepción del bien con rasgos comunitarios, como opuestos a individualistas.

Otro buen ejemplo para ilustrar esta crítica es el del patriotismo, conocidamente discutido por MacIntyre. El patriotismo, como actitud

${ }^{10}$ Sandel (1982), pág. 33.

${ }^{11}$ Sandel (1982) pág. 34.

${ }^{12}$ La cita es de Hume, en Sandel (1982) pág. 32. 
de lealtad incondicional hacia una comunidad o nación, sólo puede surgir en la medida en que se cumplan determinadas condiciones. Debo sentir un tipo especial de agradecimiento o deuda hacia mi nación, pero no por haber recibido de ella algún beneficio concreto, sino por tratarse de $m i$ nación ${ }^{13}$. Este vínculo con mi comunidad o nación sólo puede establecerse en la medida en que ella tenga, para mí, un valor superior, al punto que mi propia vida deja de tener sentido sin su existencia (de ahí la disposición a sacrificarla por ella). Esta valoración surge, a su vez, de la creencia de que la propia nación posee una misión histórica particular que cumplir, que es lo que, precisamente, da sentido a mi existencia. Hasta qué punto son necesarias todas estas condiciones para generar vínculos patrióticos, es algo discutible; pero lo que en todo caso es indudable, es que una sociedad liberal no cumple con ninguna de ellas. Las sociedades liberales (al menos de acuerdo con la teoría liberal) se limitan a crear cierto marco de reglas que permitan a los individuos perseguir fines diferentes; no hay ninguna «misión» que cumplir, ni ningún fin unificador. Por lo tanto, el liberalismo, según esta segunda crítica, no sólo es reprochable porque defiende una concepción del bien particular sin admitirlo, sino porque esa concepción del bien es tal que no permite el surgimiento de los lazos comunitarios necesarios para constituir agentes morales y hacer surgir verdaderas virtudes morales, de las cuales el patriotismo es un ejemplo, entre otros.

De acuerdo con cuál sea la posición que se tome frente a estas dos críticas, parece posible distinguir dos tipos de comunitarismo. En primer lugar, los comunitaristas que llamaré «débiles», es decir, aquellos que defienden sólo la primera crítica, y consideran compatible, por ejemplo, ser comunitaristas y ser liberales, si es que el propio marco está constituido por los valores liberales sustantivos. El liberalismo como teoría sustantiva, y como práctica política, contrariamente a lo que sostiene la teoría liberal metaética, constituye, en opinión de los comunitaristas débiles, una tradición y una concepción del bien como cualquiera. Además, en gran parte, es el marco comunitario de los propios comunitaristas. De allí que no deseen abandonar sus principios básicos, y, como dice Walzer, se vean «capturados» por su vocabulario. En segundo lugar, tenemos los comunitaristas que denominaré «fuertes», que defienden ambas críticas, subrayando fundamentalmente la segunda, es decir, aquellos que consideran que una

${ }^{13}$ MacIntyre (1984), pág. 86. 
verdadera tradición requiere algo más fuerte que lo que el liberalismo ofrece. Estos están en desacuerdo con el liberalismo incluso en cuanto al contenido de los valores liberales sustantivos, por considerar que no crean los lazos suficientes para que surjan verdaderas virtudes morales. Analicemos ahora más de cerca ambos tipos de comunitarismo:

1. El comunitarista débil sostiene, como vimos, una posición relativamente modesta. Él no defiende determinados valores, en contraposición con los valores liberales, sino que subraya solamente la vinculación necesaria entre los valores constitutivos del yo moral, y los valores del entorno o marco al cual ese yo pertenece. Este marco es constitutivo, en el sentido de que determina la concepción del bien y los fines últimos de cada individuo. Este marco puede ser, naturalmente, liberal o no serlo. Si lo es, como es el caso del entorno de los propios comunitaristas, entonces dado que el marco es «inescapable», estaremos determinados a ser liberales. Si, además, no queremos engañamos, y creer que somos liberales por alguna razón universal y neutral, seremos liberales en el nivel sustantivo y, simultáneamente, comunitaristas en el plano metaético.

Ahora bien, ¿es coherente esta posición ética? Se puede ser liberal en el plano sustantivo y no serlo en el metaético, tal como he definido ambos niveles? Creo que lamentablemente esto no es posible. La razón básica por la cual esto no es posible es que ambos niveles no son lógicamente independientes, sino que existe entre ambos una relación profunda de implicación. Intentaré mostrar esta relación entre las tesis liberales sustantivas y la tesis metaética universalista.

Un comunitarista débil debería sostener que es posible ser liberal en el plano sustantivo (es decir, defender los derechos individuales, las libertades políticas, el individualismo, etc.), $\mathrm{y}$, simultáneamente, que estos valores sólo valen para las sociedades liberales, es decir, defender el particularismo.

Creo que un buen modo de aclarar porqué esto no es posible y en qué reside el núcleo del conflicto es remitimos a un ejemplo concreto.

Supongamos que defendemos la teoría liberal sustantiva (en alguna de sus posibles versiones). Para no ingresar en terrenos conflictivos dentro del liberalismo, tomaré un ejemplo indiscutido: la libertad religiosa.

Un comunitarista débil, en tanto es un particularista moral, debe aceptar la existencia de diferentes tradiciones, diferentes comunidades con valores diferentes, cada una encarnando un determinado ideal del bien, un determinado conjunto de fines. De modo que un comunitarista débil perteneciente a una sociedad liberal, considera que tanto derecho tiene él y su comunidad en defender sus valores liberales, como cualquier otra comunidad en defender alguna otra concepción 
del bien, por ejemplo, una comunidad que imponga un ideal de excelencia humana basado en la religión. La actitud del comunitarista débil sería de no intervención y consideraría igualmente válidos sus valores que los de esta comunidad en que, digamos, no existe la libertad religiosa y una determinada religión es impuesta coercitivamente.

Pero, por otro lado, alguien que defiende la teoría liberal sustantiva (y el comunitarista débil debe defenderla, en la medida en que pertenece a este marco) consideraría que los individuos poseen un derecho a la libertad religiosa, y que el hecho de haber nacido en el país A o B no es un dato muy relevante para determinar si un individuo posee o no un derecho. Esto es una tesis sustantiva. Un liberal nos dirá que el individuo es más importante que la nación, y una nación en donde una religión es impuesta coercitivamente es una sociedad en donde los derechos son violados y los individuos oprimidos. ¿Cuál puede ser la razón, para un liberal, por la cual la discriminación religiosa es mala en el país A y buena en el país B? Sostener esto es incompatible con la posición liberal sustantiva.

Si mi argumentación respecto del comunitarismo débil es correcta, y si el ejemplo presentado es suficientemente representativo (y creo que lo es), entonces quiere decir que existe una vinculación argumentativa profunda entre las normas sustantivas típicamente liberales y (al menos) la tesis metaética universalista: no se puede ser genuinamente liberal en el plano sustantivo sin creer, además, que las normas liberales valen independientemente de los contextos, comunidades, y naciones particulares.

2. Pasemos ahora a la posición comunitarista fuerte. Podría sostenerse que el comunitarista fuerte no posee el problema del débil. Él sostiene que un comunitarista no puede defender los valores o principios de la teoría liberal sustantiva. El comunitarista fuerte critica la teoría liberal, tanto en su nivel metaético como en el nivel sustantivo, pretendiendo sustituir los valores constitutivos de la concepción del bien liberal (individualismo, egoísmo, etc.) por una concepción del bien comunitaria (solidaridad, patriotismo, lazos comunitarios, etc.). Esto parece ser más coherente, dado que el comunitarista fuerte es un particularista moral (niega el universalismo y el neutralismo liberal) que además niega las normas liberales sustantivas. Esto parece funcionar bien, en la medida que el propio marco constitutivo sea comunitario; pero, ¿qué ocurre si el mismo es liberal? Ocurre, nuevamente, una situación muy paradójica. Por un lado, el comunitarista fuerte es un particularista moral, es decir, sostiene que el yo moral (es decir los valores y normas a las que uno adquiere) está constituido por el marco o tradición al que uno pertenece. Ésta es la crítica metaética, que el comunitarista fuerte comparte con el débil. Pero, por otro lado, 
ocurre que el propio marco puede ser liberal, como es el caso, según lo que ya hemos visto, de los propios comunitaristas. Sin embargo, el comunitarista fuerte, a diferencia del débil, rechaza este marco, rechaza estos valores individualistas y egoístas. Pero entonces, ¿de dónde proviene esta posición crítica hacia su propia comunidad? ¿Cómo es que no se ve «capturado» por el vocabulario liberal? El comunitarismo no es algo que pertenezca a su marco constitutivo; su marco es liberal. Curiosamente, el comunitarista fuerte requiere razones neutrales para rechazar determinado tipo de concepción del bien (liberal), y abrazar determinado otro tipo (comunitaria). Necesita dar razones por las cuales una sociedad liberal no promueve verdaderas virtudes y sí lo hace una sociedad comunitaria ${ }^{14}$. No puede buscar las razones en su marco comunitario, porque éste es, para su desgracia, liberal. Esta capacidad de alejarse del propio marco es, precisamente, lo que afirma la tesis metaética neutralista. Y esta capacidad es lo que el comunitarista fuerte necesita para criticar su propio marco. Nuevamente, para poder defender la posición comunitarista, es necesario abrazar una tesis profundamente anticomunitarista: la tesis de que no estamos (al menos, tan) «capturados» por nuestro marco o tradición, y que este marco no es (tan) constitutivo.

El comunitarista fuerte podría ensayar la siguiente respuesta para escapar de esta dificultad. Se trata, por lo demás, de un argumento presentado a menudo por los comunitaristas, cuando desean evitar caer en un tradicionalismo conservador. La idea es formulada por MacIntyre con claridad:

«Notice that rebellion against my identity is always one possible mode of expressing it» ${ }^{15}$.

De este modo, un comunitarista fuerte podría sostener que, cuando el comunitarismo contradice las tesis sustantivas del liberalismo, está, de algún modo, siendo fiel a su identidad liberal. Pero esta estrategia es, en realidad, lo que en términos de Popper se denomina «hipótesis $a d$ hoc». Si un modo de expresar mi identidad es negándola, entonces mi identidad admite exactamente cualquier cosa. O,

${ }^{14}$ Véase la clasificación de diferentes tipos de virtudes que realiza MacIntyre, de las cuales el tipo superior, consiste en «having an adequate sense of the traditions to which one belongs or which confront one». La afirmación de que esto es una virtud es, sin duda, una afirmación universal (MacIntyre [1985], pág. 223).

${ }^{15}$ MacIntyre (1985), pág. 221. Para la aplicación de este argumento para escapar de una posición tradicionalista conservadora, que avala cualquier tradición, véase la nota anterior. 
dicho de otro modo, cualquier cosa que yo haga o diga o exprese, podría ser interpretado como expresión de mi identidad. Aun cuando el liberalismo no es una teoría monolítica y admite diferentes versiones, y aun cuando parte del ideario liberal incluye la posibilidad de cuestionamiento y crítica, esto tiene límites precisos, fuera de los cuales no tiene sentido seguir diciendo que se defienden valores liberales.

Nuevamente es útil referirse a ejemplos concretos. Uno de los principios tradicionalmente liberales consiste en sostener que solamente existe responsabilidad moral de tipo individual. Esto se sustenta parcialmente en la creencia de que sólo los individuos son agentes morales y no existen entidades colectivas a las que se les pueda adjudicar esta cualidad. Este principio no es una fórmula vacía; es una tesis sustantiva e impregna y condiciona muchos de los juicios morales concretos. Por ejemplo, un liberal debe sostener que no existe actualmente una responsabilidad moral de la gran mayoría de los alemanes por los crímenes perpetrados por los nazis. No existe ni una responsabilidad individual (dado que no fueron autores de esos crímenes), ni colectiva (dado que no hay entidades colectivas moralmente relevantes) ${ }^{16}$. Ésta es una tesis fuerte $\mathrm{y}$, tal vez, discutible. Pero lo importante ahora es que el comunitarismo debe negar este punto de vista, y de hecho algunos comunitaristas lo hacen explícitamente ${ }^{17}$. Se trata de un desacuerdo sustantivo. Si un individuo cuya identidad está constituida por la tradición liberal niega la posición liberal en este punto, defendiendo la idea de que existe una culpa moral colectiva, entonces, es mejor sencillamente sostener que se ha apartado de los valores liberales, que ya no es liberal. Lo importante, en el fondo, no es si alguien se adjudica el rótulo de liberal o no, o quién tiene derecho a hacerlo, sino que estamos frente a dos posiciones diferentes, y que si defendemos una, no podemos defender la otra.

Nótese la similitud entre las situaciones paradójicas en que quedan ambos tipos de comunitaristas: el comunitarista débil es un particularista moral, que, dada su situación particular (liberal), debe ser universalista; y el comunitarista fuerte es un particularista moral que,

${ }^{16}$ Salvo, por supuesto, los casos de criminales todavía vivos, que son individualmente responsables por sus actos. Deseo insistir en que, en este ejemplo, me estoy refiriendo a una responsabilidad moral directa. No es, por supuesto, inconsistente con el liberalismo defender la esencia de una responsabilidad política de Alemania, en el sentido de reparar los daños producidos a las víctimas del nazismo, así como la responsabilidad moral indirecta, consistente en asumir esa responsabilidad política y contribuir para esa reparación. 220-221.

${ }^{17}$ Explícitamente rechaza esta posición, en éste y otros ejemplos, MacIntyre en (1985), págs. 
para escapar de su situación particular (que lo conminaría a ser liberal), debe también recurrir a criterios neutrales ${ }^{18}$.

Un último intento de rescatar la posición comunitarista podría consistir en enfatizar la distinción entre el universalismo y el neutra-

${ }^{18} \mathrm{Al}$ margen de la discusión de este trabajo, es interesante notar que puede establecerse una vinculación entre las dos posiciones comunitaristas analizadas y un cierto relativismo moral de tipo sociológico: nuestros juicios morales y los valores que defendemos no son universales, porque están determinados por las circunstancias sociales particulares en que estamos situados. No es posible «tomar distancia» y elaborar evaluaciones morales neutrales. (Más respecto de la relación entre comunitarismo y relativismo moral en Nino (1989), págs. 158 y ss.). Sin embargo, es necesario, a mi juicio, aclarar que el relativismo moral, independientemente de sus méritos propios, posee una ventaja sustancial respecto del comunitarismo. El relativismo moral (o al menos alguna versión de éste) no pretende construir una teoría moral. Justamente, se trata de una teoría destructiva de la moral: lo único que existe, según ella, son códigos morales diferentes, pero no existe ningún criterio para establecer la validez moral de los mismos. En el nivel metaético, el relativismo niega la posibilidad de toda fundamentación racional de las normas morales. El comunitarismo, en cambio, es una teoría ética positiva, que pretende establecer criterios de validez moral. Esto marca una diferencia fundamental. El relativismo moral, en tanto es base de una posición escéptica de la fundamentación ética, puede aceptar, junto con el liberalismo, el siguiente juicio condicional: si existe un sistema moral válido, éste debe ser universal. Sólo que el determinista escéptico niega el antecedente: pero él puede perfectamente aceptar que, en el caso de que se pudiera hablar de un sistema moral justificable, al menos algunas de sus normas deberían tener validez universal. En cambio, el comunitarismo debe negar este condicional, afirmando que es posible la existencia de códigos morales justificables que no posean ninguna norma en común. El determinismo moral niega el universalismo, pero niega también la posibilidad de justificación de normas morales; en cambio, el comunitarismo niega el universalismo, pero pretende justificar una moralidad. Para expresar crudamente con un ejemplo la diferencia, el escepticismo determinista considera que la moral democrática y la moral nazi se encuentran en el mismo nivel: aquellos cuyo contexto los ha determinado a ser demócratas creerán en la primera, y aquellos cuyo contexto los ha determinado a ser nazis, creerán en la segunda, pero ninguna de las dos puede justificarse como una moral válida. El comunitarismo, por su lado, sostiene igualmente que ambas se encuentran en el mismo nivel, y por las mismas razones, pero, además, sostiene que ambas son morales válidas. Por supuesto, un comunitarista puede escapar a esta consecuencia indeseable, diciendo que el nazismo es una desviación o aberración de la tradición alemana, mientras que la democracia es un representante genuino de su respectiva tradición. Pero esto es un recurso evidentemente $a d h o c$, que, por lo demás, requiere algún criterio universal para distinguir posiciones genuinas de aberrantes. Esta estrategia puede detectarse, por ejemplo, en el trabajo de MacIntyre sobre el patriotismo, cuando menciona el caso de la resistencia de alemanes contra el nazismo (MacIntyre, 1985, pág. 97). Vuelvo sobre este tipo de estrategias en la sección siguiente. 
lismo que he realizado al comienzo del trabajo, y defender la independencia de ambas tesis, de modo de sostener alguna versión de la tesis universalista, y solamente rechazar el neutralismo. En esta dirección se encuentra el esfuerzo de Walzer en su Tanner Lectures, y al cual me referiré brevemente a continuación.

Walzer distingue dos tipos de universalismo. Por un lado, lo que él denomina «universalismo de ley general» (covering-law universalism), y, por otro, el «universalismo reiterativo». El primero está representado arquetípicamente por el liberalismo (aunque Walzer incluye en la discusión otras teorías universalistas como el marxismo, y ciertas interpretaciones de las religiones judía y cristiana), mientras que el segundo constituye una propuesta de Walzer que podría ser compatible con ciertos valores comunitaristas, como por ejemplo, el valor de la nacionalidad.

No es mi intención exponer la compleja (y a veces no del todo clara) argumentación de Walzer, pero algunas de sus principales tesis son las siguientes. En primer lugar, y como ya fue mencionado antes, el universalismo de ley general posee una pretensión hegemónica: si lo que es justo, es idéntico en toda sociedad, entonces debe tenderse a implantar esos valores, incluso contra la voluntad y la cultura imperante en determinada nación. Esto es, por lo tanto, una vía hacia el imperialismo y el sojuzgamiento. El universalismo reiterativo, en cambio, es respetuoso de las particularidades y del camino que cada nación o comunidad elige para realizar sus propios valores. ¿Qué es lo que diferencia al universalismo reiterativo del particularismo? El particularismo, como hemos visto en el caso del comunitarismo, posee la dificultad de que es incapaz de evaluar de un modo neutral la propia u otras culturas. No es capaz, en palabras de Walzer, de hacer un "rank ordering» objetivo que jerarquice de mejor a peor diferentes comunidades o naciones. El universalismo reiterativo tampoco pretende hacer esto, dado que «the rank ordering of cultures always threatens the men and women whose culture it devalues» ${ }^{19}$. Pero sí pretende realizar un «very limited ranking». Esta jerarquización objetiva mínima (que constituye el elemento universal del «universalismo» reiterativo), no se refiere a las pautas culturales internas de una nación, sino solamente al comportamiento de una nación respecto de otras, así como la represión del surgimiento de nuevas comunidades nacionales ${ }^{20}$.

${ }^{19}$ Walzer (1990b), pág. 546.

${ }^{20}$ Walzer (1990b), pág. 551: «There is no universal model for a national culture, no covering law or set of laws that control the development of a nation. But there is a universal model for the behavior of one nation toward the others». Y más adelante (pág. 552): «...reiterative universalism operates as a constraint, ruling out policies 
El universalismo reiterativo impone, por tanto, una restricción universal. Ésta consiste en un principio de no intervención o no expansión de una nación sobre otra. Excluye aquellas concepciones de la nacionalidad que contienen el valor de «nación con una misión» universal $^{21}$. Más aún, Walzer sostiene que la noción de misión (a pesar de que la hemos visto aparecer en el contexto de la defensa que MacIntyre hace del comunitarismo) es propia del universalismo de ley general. El deseo de expansión de una nación sobre otra debe ser, normalmente, justificado por los líderes políticos y los intelectuales:

«The need to justify themselves; hence their reasons are moral reasons, which take the form -I am not sure that any other is available- of coverin-law universalism» ${ }^{22}$.

Sin intentar hacer una interpretación fiel del propósito de Walzer, podemos preguntarnos de qué modo esta posición combina ciertos componentes universalistas con otros particularistas. Esquemáticamente, la idea es la siguiente. Existen ciertos valores universales, tales como el principio «that human beings are entitled to equal respect and concern ${ }^{23}$. Pero el universalismo de ley general ha convertido este principio en una ley hegemónica, pretendiendo imponer su interpretación del mismo; lo cual, a su vez, ha sido un pretexto para someter y explotar otras culturas. Este principio, en cambio, debe interpretarse de modo reiterativo, permitiendo que cada cultura realice su interpretación y recorra su propio camino, de acuerdo con sus circunstancias y su cultura. Esto 1leva, concluye el argumento, a un respeto por la particularidad, en el sentido de que el único modo de experimentar y realizar esos valores es a través de la propia cultura o nación. En definitiva, el respeto por la particularidad y el nacionalismo (no expansionista) son el camino más seguro y genuino de alcanzar esos valores universales.

Creo que este argumento admite dos interpretaciones:

1. La primera puede formularse del siguiente modo. El universalismo de ley general (especialmente liberal) y el universalismo reiterativo poseen, formalmente, los mismos ideales o principios ${ }^{24}$. Pero el

that are inconsistent with the further "development of native talent" and local cultures» (La frase citada pertenece a Berlin, citado en pág. 551

${ }^{21}$ Walzer (1990b), pág. 540.

${ }^{22}$ Walzer (1990b), pág. 539.

${ }^{23}$ Walzer (1990b), pág. 529.

${ }^{24}$ De hecho el principio citado en la nota anterior pertenece a Dworkin. 
camino para que esos ideales se realicen es diferente. El universalismo de ley general, queriendo imponer esos ideales, los frustra, al no permitir que se desarrollen espontáneamente sobre la base de las diferencias que caracterizan cada cultura. Ahora bien, esta interpretación se funda en una premisa fáctica, que, por otro lado, Walzer se esfuerza por demostrar: que el universalismo de ley general ha sido la principal fuente de imperialismo y opresión, mientras que los movimientos de liberación son de carácter nacionalista ${ }^{25}$. Más allá de la discusión acerca de si esto es empíricamente el caso $^{26}$, es claro que, en un sentido profundo, la posición de Walzer (bajo esta interpretación) no se distingue sustancialmente de la posición liberal. Se trata únicamente de una diferencia de estrategia para realizar valores comunes. De hecho, es un tema controvertido dentro del liberalismo, si es éticamente justificable o no la intervención en otros Estados para imponer coercitivamente, por ejemplo, el sistema democrático, o liberarlo de una dictadura ${ }^{27}$.

2. La segunda interpretación puede reconstruirse del siguiente modo. No se trata de una cuestión empírica o estratégica, sino conceptual. El universalismo liberal (como cualquier universalismo de ley general) posee un ideal de justicia único. Él cree poseer la interpretación adecuada del ideal de igual respeto y consideración. Pero tal interpretación única no existe. Cada sociedad debe reiterar (siempre de modo diferente y particular) la búsqueda e interpretación de ese ideal. Por ello, el único elemento universalista debe consistir en el

${ }^{25}$ Véase esp. Walzer (1990b), págs. 536 y ss.

${ }^{26}$ Dicho sea de paso, creo que es empíricamente falso. Es falso que solamente (o principalmente) doctrinas universalistas han aumentado el imperialismo, la expansión y la opresión de otros pueblos. No haré un recuerdo, pero si hemos de ser imparciales, debemos reconocer que todo tipo de doctrinas han servido como pretexto para la agresión y dominación. No solamente la de que había que llevar la civilización a pueblos bárbaros (en el caso del liberalismo), o la liberación a los pueblos oprimidos (en el caso del marxismo), sino también la que habla del «espacio vital», o, más recientemente, de la «limpieza racial» de la propia nación. Cuál de estos pretextos ha llevado a aberraciones mayores, es algo en lo que vale la pena entrar, pero debemos admitir que ninguna tiene el privilegio de la exclusividad. Por otro lado, es falso que los movimientos de liberación frente a la opresión y el imperialismo hayan sido siempre (o principalmente) de índole nacionalista. Cabe recordar (y esto lo reconoce el propio Walzer) que los valores liberales, al menos en su versión democrática, también están en contra de la opresión y a favor del derecho de autodeterminación. En resumen, no podemos abogar en favor de este o aquel valor, sobre la base de que ha sido o no usado para buenas o malas causas: todos los valores han sido utilizados para respaldar las mejores y las peores.

${ }^{27}$ Más aún, la versión liberal clásica (que puede encontrarse en Kant y Mill) rechaza todo tipo de intervención. Para una exposición de las diferentes posiciones y defensa de la justificación de la intervención en algunos casos, véase Garzón Valdés (1991). 
respeto por el «derecho a la reiteración $»^{28}$. Si bien esta segunda interpretación parece más plausible (y, probablemente, más cercana a la intención de Walzer), es todavía muy ambigua. Por un lado, entendiendo «reiteración» en un sentido más o menos estricto, debemos afirmar que el derecho a la reiteración no puede ser interpretado como un derecho a la total autodeterminación, sea cual fuere el contenido. «Reiteración» implica que ciertos contenidos mínimos de aquello que es universal deben «reiterarse». En este caso, nuevamente estamos frente a una posición muy cercana al universalismo liberal. Éste tampoco niega el derecho a diferencias culturales o de elegir «a particular understanding of the good life». Por el contrario, intenta respetar esas diferencias. Sólo que establece límites precisos a esas diferencias, límites que pretenden poseer universalidad ${ }^{29}$. Ahora bien, si no incluimos determinados límites en el derecho a la reiteración, entonces quiero decir que puede darse el caso de que no se trate en absoluto de una reiteración; y entonces no se entiende por qué se usa el término reiteración, y dónde reside el elemento universalista del «universalismo reiterativo». Una respuesta a esta objeción podría ser que, permitiendo el desarrollo espontáneo de las culturas, la reiteración se producirá también espontáneamente. Sin embargo, esto es expresamente negado por Walzer: «Reiterative universalism, by contrast, makes no predictions [...] about the substance of the successive reiterations ${ }^{30}$. En este caso, entonces, el universalismo reiterativo no se distingue relevantemente del particularismo: cualquier contenido es legítimo, y el principio de igual respeto y consideración admite cualquier interpretación. Si es así, entonces las mismas críticas que en la sección II dirigí al comunitarismo pueden aplicarse aquí.

\section{Conclusión}

Si bien la posición de Walzer merecería tal vez un análisis más detallado, me gustaría sintetizar a modo de conclusión el resultado al que hemos arribado: tanto el comunitarismo débil como el comunitarismo fuerte resultan posiciones paradójicas, cuando ocurre que el

${ }^{28}$ Véase Walzer (1990b), pág. 535: «We act immorally whenever we deny to other people the warrant for what I will now call the rights of reiteration, that is, the right to act automously and the right to formattachments in accordance with a particular understanding of the good life» (subrayado mío).

${ }^{29}$ Por ejemplo, que el «particular understanding of the good life» no puede contener el derecho de los hombres a disponer libremente de las mujeres, tal como ocurre en muchas culturas.

${ }^{30}$ Walzer (1990b), pág. 547. 
agente moral que reflexiona acerca de su identidad moral y pretende comprenderla, pertenece a una práctica moral no comunitaria, sino liberal. El comunitarista débil, porque al pertenecer a un marco liberal, se ve obligado a aceptar el universalismo liberal que él desea rechazar, es decir, se ve obligado a rechazar el particularismo moral que le es propio; el comunitarista fuerte, porque necesita adoptar las tesis neutralista (y por tanto, negar que estamos «atados» a nuestra tradición) para poder criticar y rechazar la teoría liberal sustantiva. Estas paradojas se resuelven, naturalmente, en la medida en que se abandona el rechazo a la tesis universalista, tal como ocurre con el universalismo reiterativo de Walzer. Sólo que entonces es muy cuestionable si seguimos frente a una teoría genuinamente comunitarista (y si éste fuera el caso, entonces nuevamente parece caer en los problemas del comunitarismo débil o fuerte). Si es que existe un punto en común entre los diferentes comunitaristas es el énfasis en la vinculación fundamental (o incluso «trascendental» en el sentido de Kant, tal como puede encontrarse en Taylor $^{31}$ ) entre el yo moral y el marco particular al que ese yo pertenece. Esto implica, hasta donde yo puedo entender, negar la tesis verdaderamente universalista, es decir, negar que existen normas que limitan o condicionan esa vinculación «constitutiva».

Si esto último es cierto, y si las dos posiciones comunitaristas que he distinguido son suficientemente representativas (en el sentido de que cubren de modo más o menos exhaustivo el espectro posible de teorías comunitaristas), entonces llegamos a la conclusión de que el comunitarismo debe hacer frente a algunos conflictos teóricos importantes. En el fondo, se ve enfrentado a un conflicto que (no casualmente) es similar al que se ve enfrentado todo nacionalismo, cuando la propia tradición le resulta adversa. Tal vez, todas las disquisiciones que he realizado puedan ser resumidas en la siguiente frase de Borges sobre el nacionalismo argentino: «el culto por el color local es un reciente culto europeo que los nacionalistas deberían rechazar por foráneo» ${ }^{32}$.

\footnotetext{
${ }^{31}$ Taylor (1989), pág. 32.

32 Borges (1989), págs. 270.
} 


\section{BIBLIOGRAFÍA CITADA}

Borges, J. L., «El escritor argentino y la tradición», en Discusión. Obras completas. Tomo I, Barcelona, 1989.

MacIntyre, A., «Ist Patriotismus eine Iugend?, en A. Ronnet. Kommunitarismus. Eine Debatte uber die moralischen Grundlagen moderner Gesellscharten. Frankfurt, 1993. Cito MacIntyre (1984).

MacIntyre, A., After Virtue. A Study in Moral Theory. London, 2. ${ }^{a}$ ed., 1985.

Nino, C., Etica y Derechos humanos. Buenos Aires, 2. ${ }^{\text {e ed., } 1989 .}$

Sandel, M., Liberalism and the Limits of Justice. Cambridge, 1982.

Sandel, M., «The Procedural Republic and the Unencumbered Self», Political Theory 12, 1984.

Taylor, C., Sources of the Self, Cambridge, 1989. Walzer. M., «The Communitarian Critique of Liberalism». Political Theory, 18, 1, 1990. Cito [1990a].

Walzer. M., «Nation and Universe» en G. Peterson (ed.), The Tanner Lectures on Human Values. Salt Lake City, 1990. Cito (1990b). 
DOXA 17-18 (1995) 